January 21, 1999

\title{
PNNL-12033. ERRATA
}

Distribution

Subject: Errata - PNNL-12033, In Situ Void Fraction and Gas Volume in Hanford Tank 241-SY-101 as Measured with the Void Fraction Instrument

Please replace Appendix $\mathrm{G}$ with the attached correct versions in your copy of the report.

If you have any questions call me at 375-2396.

Sincerely,

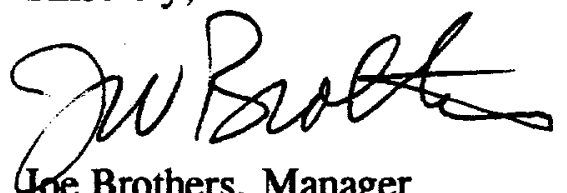

Joe Brothers, Manager

PNNL Flammable Gas Project

\section{RECEIVED \\ FEB 171999 \\ OSTI}




\section{DISCLAIMER}

This report was prepared as an account of work sponsored by an agency of the United States Government. Neither the United States Government nor any agency thereof, nor any of their employees, make any warranty, express or implied, or assumes any legal liability or responsibility for the accuracy, completeness, or usefulness of any information, apparatus, product, or process disclosed, or represents that its use would not infringe privately owned rights. Reference herein to any specific commercial product, process, or service by trade name, trademark, manufacturer, or otherwise does not necessarily constitute or imply its endorsement, recommendation, or favoring by the United States Government or any agency thereof. The views and opinions of authors expressed herein do not necessarily state or reflect those of the United States Government or any agency thereof. 


\section{DISCLAIMER}

Portions of this document may be illegible in electronic image products. Images are produced from the best available original document. 


\section{Appendix G}

\section{Gas Volume Calculation Model}

The most general expression for the gas volume contained in a cylindrical waste volume of depth, $L$, and radius, $R$, is given by the integral

$$
V_{G}=\int_{0}^{L} \int_{0}^{2 \pi} \int_{0}^{R} \beta(r, \theta, z) \pi r d r d \theta d z
$$

where $\beta$ is the local instantaneous gas indicator function (Kataoka 1986; Zhang and Prosperetti 1993). The gas indicator function is defined as

$$
\begin{array}{ll}
\beta(x)=1: & \text { gas is present at position } \mathbf{x} \\
\beta(x)=0: & \text { gas is not present. }
\end{array}
$$

The area average gas fraction, $\alpha(\mathrm{z})$, which is the fraction of the total cross-sectional area occupied by gas in the plane $z$, is defined as

$$
\alpha(z)=\frac{1}{A} \int_{0}^{2 \pi} \int_{0}^{R} \beta(r, \theta, z) \pi r d r d \theta
$$

where $A=\pi R^{2}$. The gas fraction is also often termed the 'void fraction'. Substituting Eq. (G.2) into Eq. (G.1) gives

$$
V_{G}=A \int_{0}^{L} \alpha(z) d z
$$

In evaluating the flammable gas hazard and comparing tanks, we need to adjust the insitu volume to standard pressure and temperature. Including the pressure and temperature correction in Eq. (G.1) gives the standard gas volume as

$$
\hat{\mathrm{V}}_{\mathrm{G}}=\int_{0}^{\mathrm{L}} \int_{0}^{2 \pi} \int_{0}^{\mathrm{R}} \frac{\mathrm{p}(\mathrm{r}, \theta, \mathrm{z})}{\hat{\mathrm{p}}} \frac{\hat{\mathrm{T}}}{\mathrm{T}(\mathrm{r}, \theta, \mathrm{z})} \beta(\mathrm{r}, \theta, \mathrm{z}) \pi \mathrm{rdrd} \theta \mathrm{dz}
$$

where $p(r, \theta, z)$ and $T(r, \theta, z)$ are the local pressure and temperature, respectively; $\hat{p}$ is the standard atmospheric pressure of $101,320 \mathrm{~Pa}$; and $\hat{\mathrm{T}}$ is the standard temperature, $298 \mathrm{~K}$. If we define the area average pressure, $P(z)$, and temperature, $T(z)$, in the same way as the area average gas fraction, the total gas volume at standard temperature and pressure is given by 


$$
\hat{\mathrm{V}}_{\mathrm{G}}=\mathrm{A} \int_{0}^{\mathrm{L}} \frac{\mathrm{p}(\mathrm{z})}{\hat{\mathrm{p}}} \frac{\hat{\mathrm{T}}}{\mathrm{T}(\mathrm{z})} \alpha(\mathrm{z}) \mathrm{dz}
$$

The waste is actually composed of two main layers: a floating crust and a mixed slurry layer (the loosely settled layer is assumed to be part of the slurry for the purposes of the gas volume calculation). It is also necessary to subdivide the major waste layers further into sublayers to resolve the profile of the local void fraction data and to perform the numerical analog of the integration in Eq. (G.3) and (G.5). Therefore, it is most convenient to consider each layer and sublayer separately. Performing the integration for a layer or sublayer, $i$, of thickness, $H_{i}$, and average void fraction, $\alpha_{i}$, the in situ volume, $V_{i}$, of the layer is

$$
\mathrm{V}_{\mathrm{i}}=\alpha_{\mathrm{i}} \mathrm{AH}_{\mathrm{i}}
$$

and the standard volume, $\hat{\mathrm{V}}_{\mathrm{i}}$, is

$$
\hat{V}_{i}=V_{i} \frac{p_{i}}{\hat{p}} \frac{\hat{T}}{T_{i}}
$$

where $H_{i}$ is the layer thickness, $p_{i}$ is the layer pressure, and $T_{i}$ is the layer average temperature. Because the product of pressure and temperature ratios occurs often, we define the 'effective pressure ratio,' $P_{i}$, as

$$
P_{i}=\frac{p_{i}}{\hat{p}} \frac{\hat{T}}{T_{i}}
$$

\section{G.1 Mixed Slurry Layer}

The pressure in a sublayer $i$ in the mixed slurry is calculated by

$$
p_{i}=\hat{p}+g \rho_{L} f_{S} H_{C R}+g \rho_{S L}\left(\sum_{j<i} H_{j}\left(1-\alpha_{j}\right)+\frac{1}{2} H_{i}\left(1-\alpha_{i}\right)\right)
$$

where $\rho_{\mathrm{L}}$ and $\rho_{\mathrm{SL}}$ are the liquid and degassed mixed slurry layer densities, respectively; $H_{\mathrm{CR}}$ is the crust layer thickness; $f_{S}$ is the crust submergence fraction; $H_{i}$ is the nonconvective sublayer thickness; and $\alpha_{\mathrm{i}}$ is the sublayer void fraction.

The total in situ gas volume in the mixed slurry is the sum of the gas volumes in the respective sublayers:

$$
\mathrm{V}_{\mathrm{SL}}=\mathrm{A} \sum_{\mathrm{i}=1}^{\mathrm{SL}} \alpha_{\mathrm{i}} \mathrm{H}_{\mathrm{i}}
$$


The total standard volume is similarly computed, making use of the effective pressure ratio definition of Eq. (G.8) as

$$
\hat{\mathrm{V}}_{\mathrm{SL}}=\mathrm{A} \sum_{\mathrm{i}=1}^{\mathrm{SL}} \alpha_{\mathrm{i}} \mathrm{H}_{\mathrm{i}} \mathrm{P}_{\mathrm{i}}
$$

The overall average void fraction for the entire mixed slurry layer is calculated by

$$
\alpha_{\mathrm{SL}}=\frac{1}{\mathrm{H}_{\mathrm{SL}}} \sum_{\mathrm{i}=1}^{\mathrm{SL}} \alpha_{\mathrm{i}} \mathrm{H}_{\mathrm{i}}
$$

The average pressure ratio is defined as

$$
P_{S L}=\frac{\hat{V}_{S L}}{V_{S L}}=\frac{1}{H_{S L} \alpha_{S L}} \sum_{i=1}^{S L} \alpha_{i} H_{i} P_{i}
$$

\section{G.2 Crust Layer}

The effective pressure is the hydrostatic pressure at the midpoint of the submerged portion of the crust:

$$
\mathrm{P}_{\mathrm{CR}}=\hat{\mathrm{p}}+\frac{\mathrm{g}}{2} \rho_{\mathrm{L}} \mathrm{f}_{\mathrm{S}} \mathrm{H}_{\mathrm{CR}}
$$

The in situ crust gas volume calculated from the measured void fraction and the thickness of the submerged portion of the crust. The crust submergence fraction, $\mathrm{f}_{\mathrm{s}}$, is derived from Archimedes' principle and is given by

$$
f_{S}=\frac{\frac{1}{1-\alpha_{N B}}-(1-\psi) \phi}{\frac{1}{1-\alpha_{C R}}-(1-\psi) \phi}
$$

where $\phi$ is the porosity of the material above the liquid level, and $\psi$ is its liquid saturation ( $\psi=1.0$ means the porosity is filled with liquid), $\alpha_{C R}$ is the average crust void fraction, and $\alpha_{N B}$ is the neutral buoyancy void fraction, which is computed as

$$
\alpha_{\mathrm{NB}}=1-\frac{\rho_{\mathrm{L}}}{\rho_{\mathrm{CR}}}
$$

where $\rho_{C R}$ is the ungassed bulk density of the submerged portion of the crust. We must also apply an area factor, $F_{A}$, to account for the fact that the crust occupies slightly less than the total tank area. 
The crust in situ and standard volumes are given, respectively, by

$$
\begin{aligned}
& \mathrm{V}_{\mathrm{CR}}=\mathrm{AF}_{\mathrm{A}} \alpha_{\mathrm{CR}} \mathrm{f}_{\mathrm{S}} \mathrm{H}_{\mathrm{CR}} \\
& \hat{\mathrm{V}}_{\mathrm{CR}}=\mathrm{V}_{\mathrm{CR}} \mathrm{P}_{\mathrm{CR}}
\end{aligned}
$$

The total in situ and standard gas volumes in the entire tank are the sums of the contributions of individual layers. They are given, respectively, by

$$
\begin{aligned}
& v_{G}=v_{S L}+v_{C R} \\
& \hat{v}_{G}=\hat{v}_{S L}+\hat{v}_{C R}
\end{aligned}
$$

The overall tank effective pressure ratio is computed as the ratio of standard volume to in situ volume.

$$
P_{E F F}=\frac{\hat{V}_{G}}{V_{G}}
$$

\section{G.3 Statistical Data Reduction Model}

As indicated in Eq. (G.5), the total retained gas volume in a tank at standard temperature and pressure can be calculated by integrating the product of the local void fraction, hydrostatic pressure, and waste temperature, all of which vary with elevation. The spatial variability and measurement error associated with these quantities must be included in the uncertainty of the overall retained gas volume. The multiple sources of variability and the complex calculations make it difficult to calculate the uncertainty analytically. Instead, a Monte Carlo simulation technique is used to propagate the sources of variability through numerical calculation.

There are two key elements in this statistical simulation model: performing the numerical integration for the overall retained gas volume and combining the various sources of variability to obtain the overall uncertainty using a Monte Carlo simulation technique.

With the measurements of void, density, temperature, and waste configuration (i.e., layer thickness), the numerical integration is performed by dividing the tank waste into many very thin horizontal slices. If the slices are sufficiently thin, the void, hydrostatic pressure, and temperature within a slice can be considered uniform. Values of these quantities in a slice that does not contain a measurement are estimated as that of the nearest measurement. The retained gas volume in each slice can then be calculated as the integrand in Eq. (G.5). The summation of the gas.volumes in all waste slices is a fair approximation of the integration in Eq. (G.5) for the total retained gas volume. 
The numerical integration step does not assess the uncertainty of the gas volume estimate directly. The sample measurements vary spatially and are subject to measurement error. This implies that the measurements we have are just one set of values from a distribution of all possible values. If we took another set of measurements, we would obtain different values that would give a different total gas volume estimate. If this process were repeated many times, it would produce a distribution of the total gas volume that reflects the combined impact of the uncertainties from the multiple sources.

We can simulate the possible sampling results from estimated or assumed probability distributions of the input parameters and data. These distributions need to be set based on sample data, knowledge of the physical mechanisms of gas retention, and some assumptions. To ensure a reasonable coverage for all possible combinations of void, density, temperature, and waste configuration, a large number (e.g., 5,000) of sets of measurements are simulated. The uncertainties of these derived quantities can then be estimated from the outputs of these simulations.

\section{G.3.1 Input Probability Distributions}

The following ascribes probability distributions to measurements of temperature, density, layer dimensions, and void fraction. The density and layer thickness are necessary to compute the hydrostatic pressure.

\section{Temperature}

The waste temperature is taken from MIT thermocouple measurements performed at approximately the time of the VFI tests. The temperature at each measuring elevation is assumed to be normally distributed. The measured value is taken as the mean and the standard deviation is assigned as $2^{\circ} \mathrm{C}$. This distribution, which is applied to all tanks, is assumed to reflect both nominal measurement error and lateral variability of waste temperature.

\section{Density}

Mean and standard deviations of waste densities in the mixed slurry layer are based on density measurements with the ball rheometer in 1995 (Meyer et al. 1997). The submerged crust density is assumed equal to the nonconvective layer density determined from laboratory analysis of core samples (Reynolds 1993). The densities are assumed to be normally distributed with the same distribution applied to the entire mixed slurry or crust layer.

\section{Waste Configuration}

Normal distributions are assumed for waste surface level and crust thickness. Means and standard deviations are derived by averaging several kinds of measurements.

\section{Void Fraction}

Variability of void fraction is evaluated directly from VFI void fraction measurements. This variability mainly reflects the spatial variability within a tank. The measurement error associated with individual void measurement is not included in calculation because it is small compared with spatial variability. 
The variability of void fraction is evaluated separately for the mixed slurry and crust layer. Each layer is divided into sublayers 48-cm (19-in.) thick. This thickness is chosen to match the length of an RGS segment for convenience in later combination of VFI and RGS data. This choice allows VFI and RGS data to be weighted equally when combined.

For each sublayer containing one or more void measurements, a sample mean is calculated as the average of all the measurements in the sublayer. A common lateral variability is assumed for all sublayers in nonconvective layer. This lateral variability is estimated by a pooled sample variance, which is a weighted average of sample variance from all sublayers with more than one void measurement. The pooled sample standard deviation is calculated as

$$
\hat{\sigma}_{\text {pooled }}=\sqrt{\frac{\sum_{i} n_{i} \hat{\sigma}_{i}{ }^{2}}{\sum_{i} n_{i}}}
$$

where $n_{i}$ denotes the number of void samples in sublayer $i$, and $\sigma_{i}$ denotes the sample standard deviation in sublayer $i$. The weighting emphasizes the sublayers containing the most measurements.

It is assumed that void measurements within a sub-layer are from a common normal distribution with the estimated mean void of the sub-layer and the pooled sampled standard deviation. The void measurements from the same riser are assumed to be correlated with a covariance equal to the riser to riser variability. The VFI data show that, in some tanks, void fraction measurements from one riser tend to be higher than the measurements from the other riser. The simulated sample measurements should reflect this tendency.

Void fraction measurements with this kind of riser-dependent structure can be simulated by imposing a covariance on a set of completely independent samples. Specifically, a covariance matrix is constructed with a dimension equal to the number of void fraction measurements in the main layer. The diagonal elements of the matrix are equal to the variance of the distribution from which the measurements are simulated. Because a common variance is assumed for all sublayers, all diagonal elements are all the same. The off-diagonal elements in the matrix represent covariance between two void measurements. If two measurements came from the same riser, the covariance is set equal to the riser-to-riser variability. Otherwise, the covariance is zero. Based on the properties of normal distributions, this matrix can be used to convert a set of completely independent random samples to a set of samples with the desired riser-to-riser structure. The riser to riser variability was estimated through the following Analysis of Variance (ANOVA) model.

$$
\alpha_{\mathrm{ijk}}=\bar{\alpha}+R_{i}+L_{j}+\varepsilon_{i j k}
$$

where

$$
\begin{array}{ll}
\alpha_{i j k} & =\text { local void fraction measurement } k \text { in riser } i \text { at layer } j \\
& =\text { mean void fraction in entire layer } \\
R_{i} & =\text { deviation of void fraction at riser } i \text { from the mean }
\end{array}
$$


$\mathrm{L}_{\mathrm{j}} \quad=$ deviation of the void fraction in the $\mathrm{jth}$ sublayer

$\varepsilon_{\mathrm{ijk}} \quad=$ sampling and measurement error

The riser effect $R_{1}$ is considered as a random effect with zero mean and standard deviation $\sigma_{R}$. This standard deviation, which represents the riser-to-riser variability, was estimated by fitting the model to the VFI data for each tank.

\section{G.3.2 Calculation Procedure}

To numerically integrate the void fraction measurements and to propagate the sources of variability, the waste is divided into many very thin slices. We choose a $4.8-\mathrm{cm}$ (1.9-in.) slice thickness, $1 / 10^{\text {th }}$ of the nominal $48 \mathrm{~cm}$ (19-in.) sublayer dimension. The idea is to simulate possible sample measurements (temperature, void, etc.); therefore, the waste condition in each thin slice can be evaluated from a given set of simulated measurements. Then the mean void fraction is estimated as the average void fraction in all the thin slices. The total retained gas volume is estimated as the sum of the gas volumes in each slice.

A set of measurements is simulated from the distributions described above using the following procedure:

1. Simulate a realization of waste level, crust thickness and submergence fraction, mixed slurry layer depth, and density from the predefined distributions.

2. Divide crust and mixed slurry layers into slices 1.9 -in. thick based on the current realizations of waste configuration for the purpose of numerical integration.

3. Simulate a realization of temperature at each measuring location. If a waste slice does not contain any measuring location, the temperature of the slice will be the value of the realization at nearest elevation.

4. Simulate a realization of void fraction at each sampling location. Void fraction realizations at sampling locations within the same 19-in. sublayer are simulated from the same distribution. The void fraction value for each waste slice is calculated using the same procedure as for temperature.

For each simulation, one realization of average void fractions, gas volumes and effective pressures for crust, mixed slurry, and whole tank is calculated. Using $i$ to index a waste slice, the following equations are used for the calculation: 


$$
\begin{aligned}
& \alpha_{\text {mean }}=\frac{1}{n_{\text {slice }}} \sum_{i} \alpha_{i} \\
& V_{G}=A \sum_{i} \alpha_{i} h_{i} \\
& \hat{v}=A \sum_{i} \alpha_{i} h_{i} \frac{p_{i}}{\hat{p}} \frac{\hat{T}}{T_{i}}
\end{aligned}
$$

where $n_{\text {slice }}$ is the number of slices in a layer; $h_{i}$ is the height of a slice; $\alpha_{i}, p_{i}$ and $T$ denote void, pressure, and temperature, respectively, in $\mathrm{i}^{\text {th }}$ slice. The total gas volume is the sum of the gas volumes in the two waste layers.

A set of 5,000 realizations is obtained for each quantity of interest. A summary of the output distributions, including mean, median, standard deviation, percentiles, etc., is then calculated from these distributions.

\section{G.4 References}

Kataoka I. 1986. "Local Instant Formulation of Two-Phase Flow." Int. J. Multiphase Flow, Vol. 12, pp. 745-757.

Meyer PA, ME Brewster, SA Bryan, G Chen, LR Pederson, CW Stewart, and G Terrones. 1997. Gas Retention and Release Behavior in Hanford Double Shell Waste Tanks. PNNL-11536 Rev. 1, Pacific Northwest National Laboratory, Richland, Washington.

Reynolds DA. 1993. Tank 101-SY Window E Core Sample: Interpretation of Results. WHC-EP-0628, Westinghouse Hanford Company, Richland, Washington.

Zhang DZ and A Prosperetti. 1993. "Ensemble Averaged Euler Equations and Added Mass for Linearized Flows." Gas-Liquid Flows 1993. FED-Vol. 165, American Society of Mechanical Engineers, New York. 